\title{
Detection of Retinal Blood Vessels from Ophthalmoscope Images Using Morphological Approach
}

\author{
Jyotiprava Dash* and Nilamani Bhoi+ \\ *Department of Electronics \& Telecommunication, VSSUT, Burla, India \\ +Department of Electronics \& Telecommunication, VSSUT, Burla, India \\ Email: jyotipravadash89@gmail.com*, nilamanib@gmail.com+
}

Received 29th Apr 2016; accepted 5th Feb 2017

\begin{abstract}
Accurate segmentation of retinal blood vessels is an essential task for diagnosis of various pathological disorders. In this paper, a novel method has been introduced for segmenting retinal blood vessels which involves pre-processing, segmentation and post-processing. The pre-processing stage enhanced the image using contrast limited adaptive histogram equalization and 2D Gabor wavelet. The enhanced image is segmented using geodesic operators and a final segmentation output is obtained by applying a post-processing stage that involves hole filling and removal of isolated pixels. The performance of the proposed method is evaluated on the publicly available Digital retinal images for vessel extraction (DRIVE) and High-resolution fundus (HRF) databases using five different measurements and experimental analysis shows that the proposed method reach an average accuracy of 0.9541 on DRIVE database and $0.9568,0.9478$ and 0.9613 on HRF database with healthy, diabetic retinopathy (DR) and glaucomatous images respectively.
\end{abstract}

Key Words: Retinal Blood Vessels, Vessel Segmentation, Contrast Limited Adaptive Histogram Equalization, Gabor Wavelet Transform.

\section{Introduction}

The anatomy of the retinal blood vessels provides information for diagnosis and treatment of different ophthalmological disorders like diabetic retinopathy, hypertension, glaucoma, cataract, cardiovascular disease etc [1]. Changes in blood vessels leads to a principal disease termed as Diabetic Retinopathy and it is the main reason of blindness [2]. If the blood vessels are devastated then it may leak blood and grow brittle new vessels. When nerve cells are impaired vision diminishes which causes blurring of vision and hemorrhage into eye. If proper care is not done then it may cause retinal detachment. For better diagnosis and treatment of these diseases the blood vessels should be segmented properly.

In order to extract the retinal blood vessel, different algorithms have been proposed which can be partitioned into five main groups: matched filtering, mathematical morphology, vessel tracking, supervised learning and unsupervised methods. The matched filtering is the most extensively used algorithm for segmentation of retinal

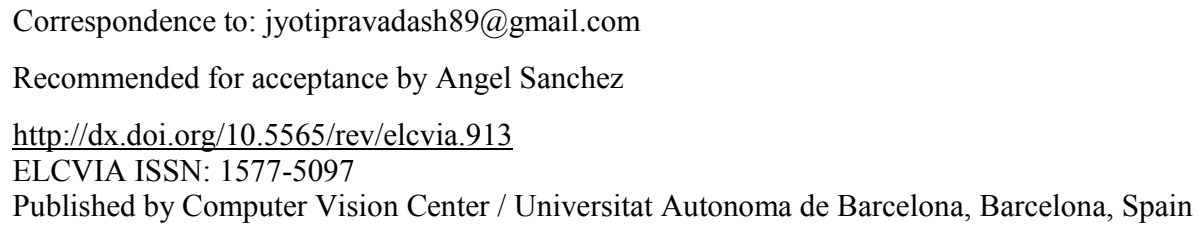


blood vessels which is initially introduced by Chaudhuri et al. [3]. In this method a former assumption is made that the cross section of the vessels can be proximated by a Gaussian function. Al-Rawi et al. [4] proposed an algorithm for automatic extraction of blood vessels by optimizing the parameters of the matched filter using genetic algorithm. Matched filter with first order derivative of Gaussian has been elaborated in [5], where the blood vessels are identified by thresholding the retinal image's response to the matched filter and the threshold is adjusted by image response's to the first order derivative of Gaussian. Cinsdikici et al. [6] applied a hybrid method using ant colony and matched filter to extract blood vessels which improve the performance accuracy of the resultant images. In [7], the authors proposed an improved multiscale matched filter using PSO algorithm where first the multiscale matched filter improve the quality of the vessels then the optimization algorithm is used to optimize the parameters of the multiscale matched filter and hence improve its performance.

Morphological image processing is a gathering of non-linear processes linked to the shape or morphology of features in an image. Morphological operations apply a structuring element to an input image, producing an output image of the same size. In [8], the segmentation of blood vessels in the eye fundus images is rely on morphological and topological analysis. Initially the morphological filter enhances the blood vessels and attribute images are created from a combination of top-hat transform with linear filter at two different scales which leads a complementary information. In the next phase linear structure are extracted using path opening filters and finally the segmented blood vessels are obtained by fusion step and automatic thresholding. In [9], Budai et al. proposed an approach which utilizes an algorithm based on vessel enhancement method combined with multiresolution framework to decrease the computational needs and to increase the sensitivity. In [10], the author illustrated an automatic retinal blood vessels segmentation method with trainable COSFIRE filter that responds to some selected vessels. By utilizing the local information vessel tracking algorithm segments the vessel between two points and the main advantage of this method is that it gives information about individual vessel rather than the entire vasculature structure [11]. In [12], the author introduced a tracking process which place on adaptive exploratory processing at the image gray level. Delibasis et al. in [13] introduced an automatic model based tracing algorithm which segments the vessels and approximates the central axis and diameter of the vessel. Supervised learning is the machine learning task of hypothesized a function from labeled training data [14]. The training data includes a set of training examples where each example is a pair comprising an input object and a desired output value. The supervised method examines the training data and generate an inferred function which further used for mapping new examples. Soares et al. in [15] introduced a supervised method using 2D Gabor wavelet and supervised classification where the method produces segmentations by classifying each image pixel as vessel or nonvessel, based on the pixel's feature vector. In [16] Staal et al. applied an automatic extraction algorithm which extracts the image ridges to form line elements that divides the image into a number of patches. After this feature vectors of each pixel are calculated. In the final stage of the algorithm, K nearest neighbors (KNN) classifier and sequential forward feature selection method is used for classification of features. The unsupervised learning is a class of machine learning that extracts conclusions from data sets which comprises input data without labeled responses and the data are clustered into different classes. In [1], the author proposed a method for vessel segmentation using level set and region growing, where initially a processing step is carried out where the image is enhanced using contrast limited adaptive histogram equalization and 2D Gabor filter. Then the enhanced image is smoothed and its boundary is preserved using the anisotropic diffusion filter. Finally the vessels are extracted using region growing and region based active contour with level set. A spatially weighted fuzzy c-means clustering based thresholding is utilized by the author in [17], where first the vessel enhancement is completed by the matched filtering followed by the extraction of blood vessels using FCM based threshold.

In spite of the fact that, many researchers have carried out their research work in retinal blood vessels extraction and obtained good performance measures, still there are certain consequences such as pixels disconnectivity, identification of thin vessels which are required to be fixed. So in this paper a morphological approach has been introduced for detection of retinal blood vessels which includes: pre-processing, segmentation and post-processing. In the pre-processing stage the input image is enhanced using contrast limited adaptive histogram equalization (CLAHE) [18] followed by 2D Gabor filter. Then the blood vessels are extracted from the enhanced image using a morphological reconstruction approach. Further a post-processing phase of two steps: (i) gap filling and (ii) removal of falsely detected isolated pixels are applied for extraction of retinal blood vessels. In 
this paper we have taken a new high resolution fundus database for performance evaluation. Our method has the advantage that it has lower computational complexity and running time of the process is low.

The paper is lined up as follows, Section 2 includes the pre-processing of the ophthalmoscope images and segmentation method. Section 3 elaborates the experimental evaluation and comparisons. Finally, the paper is concluded in Section 4.

\section{The Proposed Method}

Fig. 1 shows the block diagram of the proposed method in which the green channel image is enhanced using CLAHE and 2D Gabor wavelet. The segmentation task is performed on the enhanced image where the blood vessels are separated from background. Along with segmented blood vessels some isolated pixels are found to be distributed throughout the image which can be removed in the segmented post-processing step.

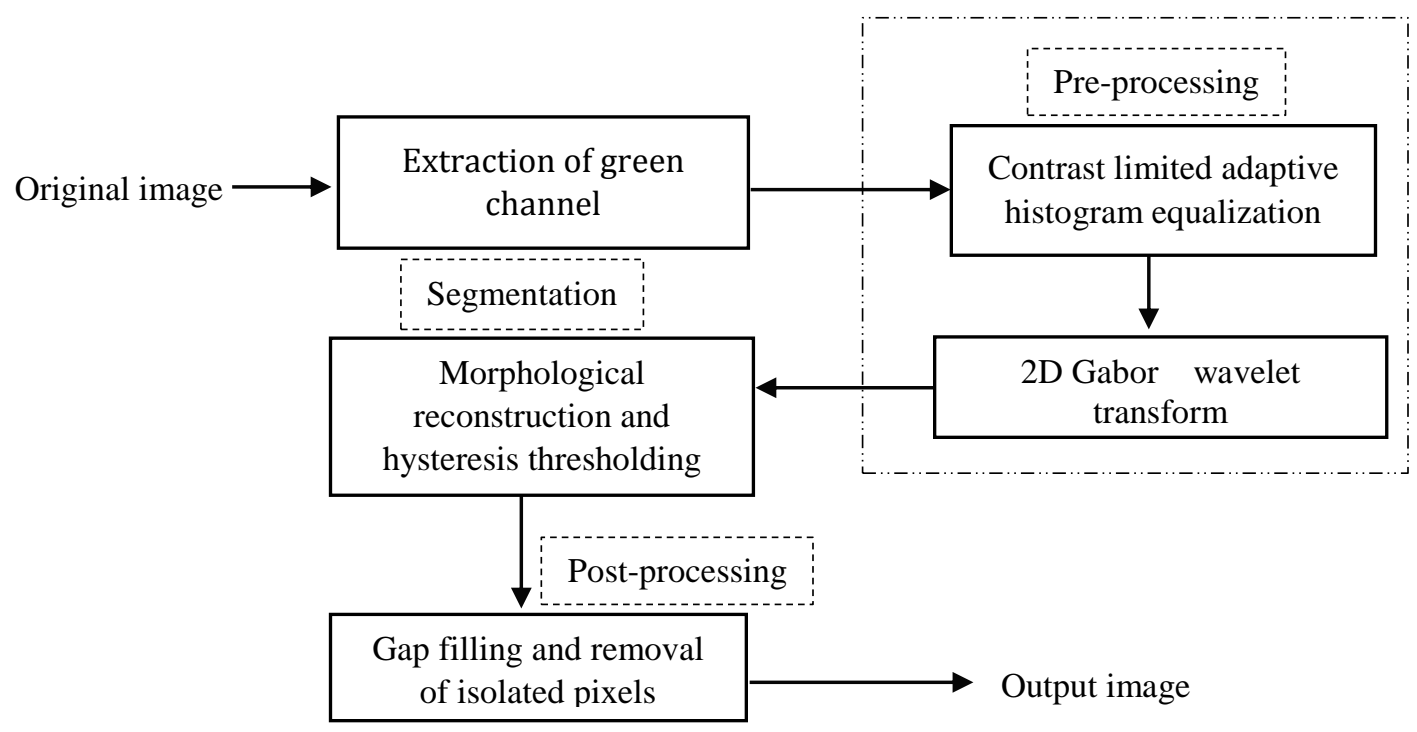

Figure 1: Block diagram of the proposed method.

\subsection{Pre-processing}

At first the original fundus RGB image is taken and its corresponding red, green and blue channels are extracted and green channel is taken as it exhibits best contrast as compared to red and blue channel. The pre-processing stage comprises following steps: contrast limited adaptive histogram equalization (CLAHE) to adjust the non-uniform illumination followed by Gabor wavelet transform to further enhance the image. Fig. 2 shows the original image and its corresponding extracted channel image, output of the contrast limited adaptive histogram equalization and the enhanced image after passing through the Gabor wavelet transform respectively.

\subsubsection{Contrast limited adaptive histogram equalization (CLAHE)}

Contrast limited adaptive histogram equalization (CLAHE) is a variant of adaptive histogram equalization (AHE). The AHE is an image enhancement technique which is used to boost the contrast of the images. The AHE is used to get the mapping for each pixel rely on its local intensity spreading. This method is therefore appropriate for enhancing the local contrast but it has a tendency to over amplify the noise in relatively homogeneous regions 
of an image [19]. So CLAHE is used to overcome this problem. CLAHE splits the entire image into a number of tiny regions of equal size and operates on each region where contrast of each small region is enhanced so that histogram of the output image matches with the histogram specified by the distribution parameter. The neighboring small regions are then combined using bilinear interpolation that remove the artificially induced boundaries. The over amplification of noise can be avoided by limiting the contrast of the individual homogeneous region. Here we have taken uniform distribution with clip limit 0.01 .

\subsubsection{Two dimensional Gabor wavelet transform}

Gabor filter is entitled after Dennis Gabor which is a linear filter use for edge detection and predominantly suitable for texture representation and discrimination. In the spatial domain, a two dimensional Gabor filter is a Gaussian function modulated by a sinusoidal plane wave [20]. This is used due to its directional selectiveness ability of distinguishing oriented characteristics and fine tuning to precise frequencies [21]. Gabor function provides optimal resolution in both spatial and frequency domain. It has both multi-resolution and multiorientation properties. The Fourier transform of a Gabor filter's impulse response is the convolution of the Fourier transform of the harmonic function and the Fourier transform of the Gaussian function in accordance with the convolution theorem $[22,23]$. The Gabor wavelet transform can be selected as the analyzing wavelet. The Gabor filter can be expressed as [24],

$\psi G(x)=\exp \left(j f_{0} x\right) \exp \left(-\frac{1}{2}|D x|^{2}\right)$

Where,

$j^{2}=-1, f_{0}$ defines the frequency of the signal and taken as $[0,2.8]$ as it gives suitable Gabor response and highest contrast, $D$ is a $2 \times 2$ diagonal matrix which can be represented as,

$$
D=\operatorname{diag}\left[\varepsilon^{-1 / 2}, 1\right], \varepsilon \geq 1
$$

Where, $\varepsilon$ parameter is critical as its higher value gives more wide width and smaller value gives less enhancement effect. So it is taken as 3. For each pixel maximum response overall direction is evaluated and the result of the Gabor wavelet can be defined as,

$$
M_{\psi}(b, a)=\max \theta\left|T_{\psi}(b, \theta, a)\right|
$$

Where,

$$
\mathrm{T}_{\psi}(\mathrm{b}, \theta, \mathrm{a})=\mathrm{C}_{\psi}{ }^{-1 / 2} \mathrm{a} \int \exp (\mathrm{jfb}) \widehat{\psi}^{*}\left(\mathrm{ar}_{-\theta} \mathrm{k}\right) \hat{\mathrm{e}}(\mathrm{k}) \mathrm{d}^{2} \mathrm{k}
$$

Where $C_{\psi}$ is the normalizing constant, $\psi$ is analyzing wavelet, a is the dilation scale and taken as 2.8 as this value can preserve shape of most vessels, $\mathrm{b}$ is the displacement vector, $\theta$ is the angle of orientation ranging from $0^{\circ}$ to $170^{\circ}$ and $\mathrm{e}$ is the finite energy. Hence it enhances the contrast of the retinal image. It can be perceived that the Gabor filter offers more enhanced image as compared to other image enhancement technique.

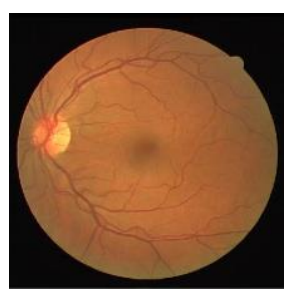

(a)

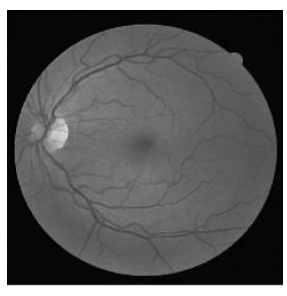

(b)

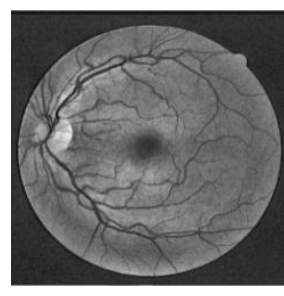

(c)

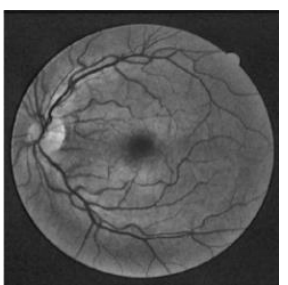

(d)

Figure 2: (a) Original image, (b) green channel image, (c) contrast limited adaptive histogram equalization enhanced image, (d) Gabor wavelet output. 


\subsection{Segmentation}

Morphological image processing is the process of extracting image features whose shape is known prior [25]. Application of mathematical morphology results in elimination of much of the small details which can be retrieved by utilizing mathematical reconstruction approach. The morphological reconstruction method is based on a source or mask image that is the reference image used in the operation, a marker image which is created based on the characteristics of the mask image with the help of a linear structuring element and a marker point, which is the beginning point for initiation of the process. The mask should be chosen carefully as it restricts the result after dilation and erosion. In the first stage reconstruction by dilation is accomplished which can be defined as carrying out iterative geodesic dilation defined by equation (5) and repeats until no changes will occur. Instead of using structuring elements it uses connectivity. Reconstruction by dilation applied on the image and reconstructs bright regions. A starting point is chosen and the pixels are reconstructed by distributing the illumination value. It begins with the maximal gray valued pixel of the marker image and reconstructs the neighboring pixels ranging from 0 to maximal valued pixel. Let $\mathrm{F}$ and $\mathrm{G}$ the marker and mask image respectively, and $\mathrm{B}$ a linear structuring element. Mathematically reconstruction by dilation can be expressed as,

$I_{D}=R_{G}^{D}(F)=D_{G}{ }^{K}(F)$

Where,

$D_{G}{ }^{1}(F)=(F \oplus B) \cap G$

$R=$ denotes the reconstruction operation, $k=$ Number of iterations, $I_{D}=$ Dilated image

Reconstruction by dilation results in exclusion of bright structures smaller than structuring element without altering the shape and from the preserved features, reconstructs connected components [26]. This operation yield an image $\mathrm{I}_{\mathrm{D}}$ that contains background. After that reconstruction by opening is performed which can be expressed as,

$I_{O}=D_{R}(E(F), F)$

Where,

$D_{R}=$ Dilation Reconstruction, $E=$ Erosion

Morphological opening is the erosion followed by dilation where erosion opens dark holes and dilation recover shapes and removes dark features smaller than the structuring elements and gives output image $I_{O}$ which contains both vessels and background. Subtraction of $I_{D}$ from $I_{O}$ gives rise a new image which includes only the vessels [27]. After this the blood vessels are brightened by calculating the second order derivative of the smoothed signal which is achieved by convolving the Gaussian filter with the signal along the vessel cross-sectional direction and vessel centerline direction which gives the output . But due to deviations in the illumination the center of the larger vessels looks darker than the edges of the vessels. So in order to overcome this problem again reconstruction by dilation is performed by taking the output of the second order derivative $I_{S D}$. Then reconstruction by erosion which reconstructs the dark regions in the image. This operation starts with the minimum valued pixel of the marker image and reconstructs the neighboring pixels ranging from minimum valued pixel to the maximum value of the image and removes the bright features smaller than the structuring. Mathematically it can be expressed as,

$I_{E}=R_{G}{ }^{E}(F)=E_{G}{ }^{k}\left(I_{S D}\right)$

Where,

$I_{E}=$ Eroded image, $I_{S D}=$ Output of the second order derivative

$E_{G}{ }^{1}=\left(I_{S D} \ominus B\right) \cup G$

Fig. 3 represents the output of morphological reconstruction. This output is undergoes hysteresis thresholding which is computed using a low and a high threshold value $T_{l}$ and $T_{h}$ respectively. In hysteresis thresholding, any pixel with intensity value above $T_{h}$ are set to 1 and any pixel with intensity value below $T_{l}$ are set to 0 . Pixels that have intensity value above $T_{l}$ and below $T_{h}$ are set to 1 if the 
connectivity is 1 . Applying hysteresis thresholding two binary images are created that is $I_{l}$ and $I_{h}$. $I_{l}$ is created by thresholding $I_{E}$ with $T_{l}$ and $I_{h}$ is created by thresholding $I_{E}$ with $T_{h}$, after that $I_{h}$ is reconstructed into $I_{l}$ which can be expressed as,

$$
\text { Hyst }_{T}(I)=R_{T_{t h}(I)}\left(T_{t h(I)}\right)
$$

$T$ denotes threshold transformation. The final segmented image is obtained by hysteresis thresholding $I_{E}$ with suitable values of $T_{l}$ and $T_{h}$. In our work we have taken the value of $T_{l}=T_{h}=T h=35$,

$$
I_{H}=\operatorname{Hyst}_{\left(T_{l}, T_{h}\right)}\left(I_{E}\right)
$$

The threshold values are selected such that it can give a balanced performance for both sensitivity and specificity. The selection of threshold values affects the sensitivity and specificity which finally affects the accuracy of the segmentation process. If thresholding is low then sensitivity is high and specificity is low that means more vessels are correctly identified as vessels and false vessel detection rate increases. For selection of threshold we have plotted a graph between threshold and accuracy where $\mathrm{x}$-axis represents the threshold values and y-axis represents different values of accuracy for each threshold in the x-axis. From the graph, we can observed that different values of threshold are taken from 15-55 and accuracy is calculated for each threshold values. We can see that for threshold values 15 to 35 it starts increasing and after 35 it begins to decreases. So 35 is the optimal point where we are getting maximum value of accuracy. We have to take the threshold value such that it can identify more vessels and from that we can remove the nonvessel in the later stage by applying morphological operators. Fig. 4 shows the variation of accuracy with respect to the different value of threshold.

\subsection{Post-processing}

The ultimate segmentation outcome is gained by the insertion of a two-step post-processing steps using morphological operations: the foremost step is the hole filling among pixels in the identified blood vessels and subsequent step is elimination of falsely detected isolated pixels. After the segmentation output obtained we can observed that the output image loses connectivity among some pixels and some nonvessel pixels are mistaken as vessel pixels which can be solved by application of morphological operators. From the obtained segmented output we can observed that the vessels may have some gaps which can be filled by using morphological filling operation that fill some holes in the obtained binary image. Taking 8-connectivity into consideration the filling operation consider that the pixels with at least eight neighbors classified as vessel points are vessel pixels. The result contains some isolated regions which are misclassified as vessel pixels [28]. These misclassification can be removed by calculating the area of each connected region and considering that if the area is less than 25 then it can be marked as nonvessel and removed. The images before and after applying post-processing are depicted in Fig. 5. Fig. 6 shows the original image of DRIVE database and its corresponding extracted blood vessels respectively.

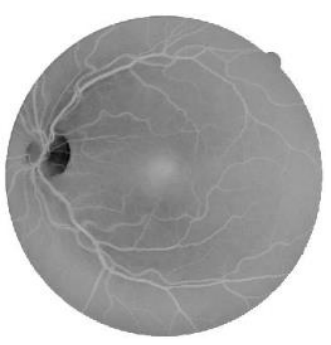

a

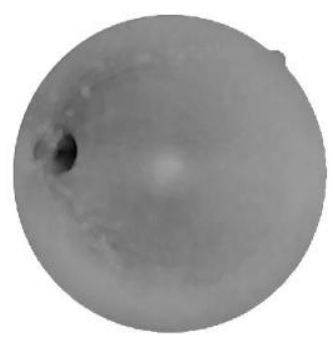

$\mathrm{b}$

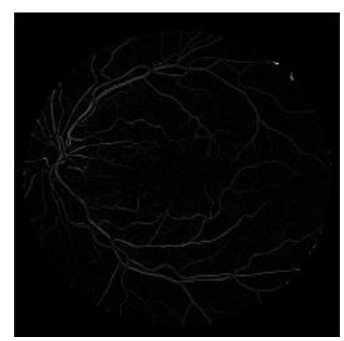

$\mathrm{C}$

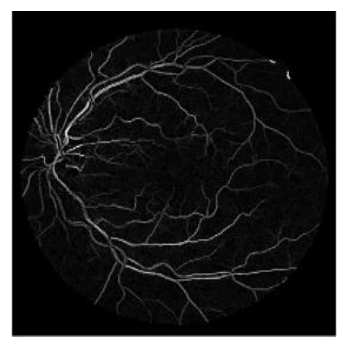

d

Figure 3: Output of morphological operation: (a) reconstruction by dilation, (b) opening, (c) difference image between dilation and opening, (d) reconstruction by erosion. 
Dash. J. et al. / Electronic Letters on Computer Vision and Analysis 16(1):1-14; 2017

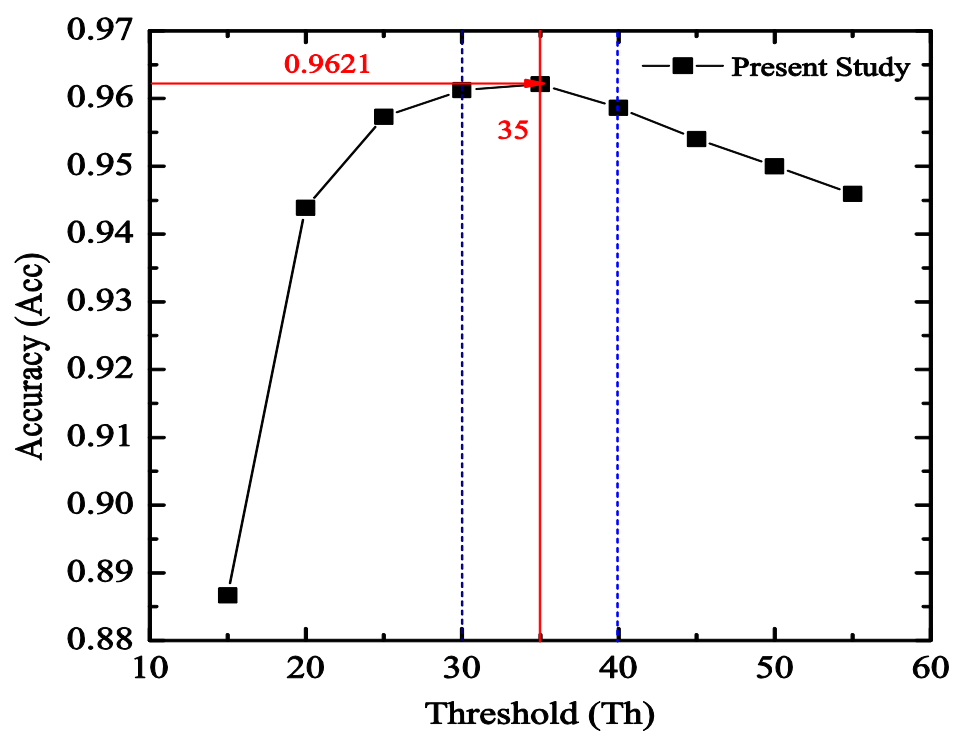

Figure 4: Variation of accuracy with respect to threshold.

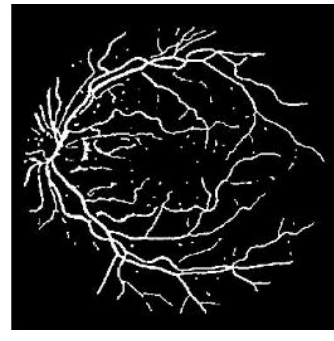

(a)

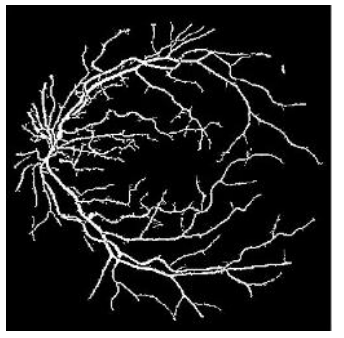

(b)

Figure 5: (a) Image before post-processing (b) Image after post-processing.

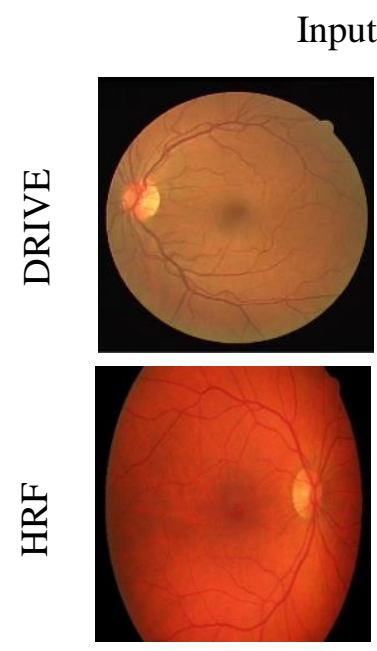

Input images
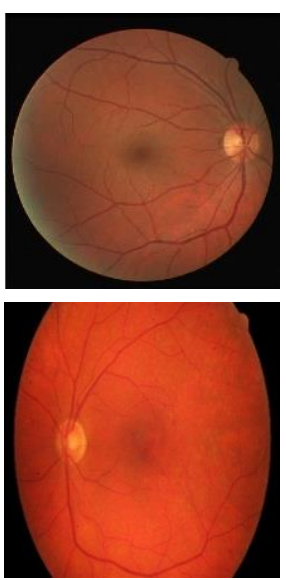

Segmented images
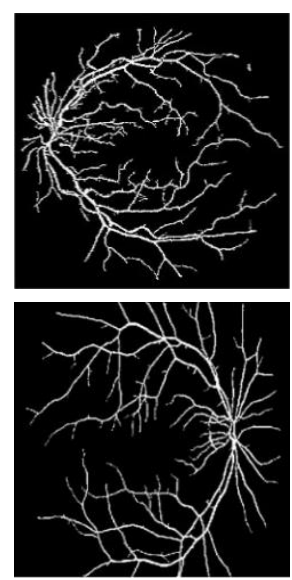
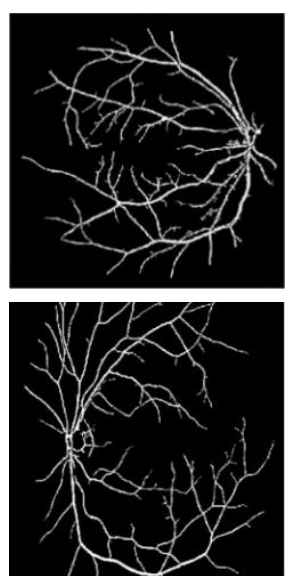

Figure 6: Segmentation results for DRIVE and HRF databases. 


\section{Experimental Evaluation and Comparisons}

The staging of the segmentation method is verified and estimated with the help of two publicly available DRIVE (Digital retinal images for vessel extraction) and HRF (High resolution fundus) databases.

In DRIVE data base [29] a canon CR5 non-mydriatic 3CCD camera with a $45^{\circ}$ field of view (FOV) is used to acquire images where each image was captured using 8 bits per color plane at $584 \times 565$ pixels. The FOV of each image is circular with a diameter of approximately 540 pixels where the images have been cropped around the FOV. For each image the mask is available that portrays the FOV. The database contains a total of 40 images which can be divided into a training and a test set where each of them includes 20 images where 7 images contain pathology. Here the images are JPEG compressed. The HRF database [30] contains 15 images of healthy patients, 15 images with diabetic retinopathy and 15 images with glaucoma. These images are captured using CANON CF$60 \mathrm{UVi}$ camera with a FOV of $45^{\circ}$ and different acquisition setting with a resolution of $3504 \times 2336$ pixels. Manual segmentation results for all the images and mask for the FOV for particular images are provided by different experts. Here the size of the images are resized in to $584 \times 565$.

The performance of the proposed method is evaluated by comparing the segmented image with the ground truth image. The ground truth image can be achieved by generating a vessel mask manually where all the vessel pixels are set to one while non-vessel pixels are set to zero. As the pixels are classified either as vessel or nonvessel so four different possibilities can be achieved like true positive (TP), true negative (TN), false negative ( $F N)$ and false positive $(F P)$ [11]. If the pixels are identified as vessels in both the ground truth and segmented image then the classification is termed as true positive. True negative when the pixels are classified as non-vessel in both ground truth and segmented image. When a pixel is identified as non-vessel in segmented image but as vessel in ground truth image, it is called as false negative. The false positive is defined as when a pixel is marked as vessel in segmented image and non-vessel in ground truth image. The comparison of different blood vessel segmentation methods with the proposed method taking the first image from the DRIVE database are shown in Fig. 4.The results of the proposed method is compared with Zhao et al. [1], Zhang et al. [5], Soares et al. [15], Cinsdikici et al. [6], Xinge You et al. [31], Budai et al. [9], Azzopardi et al. [10] and Odstrcilik et al. [32]. The level set and region growing method [1] gives poor segmentation result for some abnormal retinal images, in [5], the MF-FDOG method loses connectivity and includes some unwanted structure. Drawback of 2D Gabor filter and supervised classification method [14] is that the thin vessels are not correctly identified while in the MF/Ant method [6] after segmentation the vessels become thicker as compared to the ground truth image and in the method proposed by Xinge You et al. [31] some thin vessels are over estimated due to vessel like noise. The results of HRF database which includes healthy images, diabetic retinopathy images and glaucomatous images are compared with the improved matched filtering method proposed by Odstrcilik et al. [32], Budai et al. [9] and Frangi et al. [33]. Fig. 6 shows the comparison of image of each data set that is DRIVE and HRF (healthy, diabetic retinopathy, glaucomatous) with its corresponding ground truth image. Fig. 7 shows the comparison proposed method with method proposed by $[1,7,15]$ taking the first image from the DRIVE database. In this paper five different mathematical measures such as sensitivity $(S n)$, specificity $(S p)$, positive predictive value $(P P V)$, negative predictive value $(N P V)$ and accuracy $(A c c)$ are used for experimentation [34]. Sensitivity can be defined as the ability of the segmentation method to identify the number of pixels as vessel pixels. Specificity is the measure of ability of the segmentation technique to mark non-vessel pixels. Accuracy is the measure of ability to identify the degree of conformity of the segmented image to the ground truth image. Positive predictive value can be defined as the ratio of the pixels correctly classified as vessels to the total number of pixels classified as vessels. Negative predictive value can be defined as the ratio of pixels classified as background pixels that are classified correctly [27].

Mathematically these parameters are expressed as given in Table 1. The segmentation performances are shown in Tables 2 for DRIVE database and Table 3-5 for HRF (images of healthy, diabetic retinopathy and glaucomatous retinas) database respectively. Table 6 and 7 represents the comparison of performance of the proposed technique with other existing techniques on DRIVE and HRF databases respectively. From Table 5 and 6 , it can be marked that the proposed method outperforms the other methods by giving high accuracy and specificity in the DRIVE 
database and in HRF database it outperforms the method proposed by $[32,33]$ and the method proposed by Budai et al. [9] gives more accuracy than our method. The compared existing methods are taken from its original literatures.

\begin{tabular}{|cc|}
\hline Measure & Expression \\
\hline$S e$ & $T P /(T P+F N)$ \\
$S p$ & $T N /(T N+F P)$ \\
$P P V$ & $T P /(T P+F P)$ \\
$N P V$ & $T N /(T N+F N)$ \\
$A c c$ & $(T P+T N) /(T P+F N+T N+F P)$ \\
\hline
\end{tabular}

Table 1: Performance metrics for retinal blood vessel extraction

\begin{tabular}{|cccccc|}
\hline Image & $S e$ & $S p$ & $P P V$ & $N P V$ & $A c c$ \\
\hline 1 & 0.7452 & 0.9798 & 0.7838 & 0.9751 & 0.9621 \\
2 & 0.6632 & 0.9874 & 0.8574 & 0.9625 & 0.9542 \\
3 & 0.6343 & 0.9936 & 0.8894 & 0.9435 & 0.9507 \\
4 & 0.6931 & 0.9996 & 0.9887 & 0.9354 & 0.9370 \\
5 & 0.7848 & 0.9976 & 0.9553 & 0.9496 & 0.9498 \\
6 & 0.5401 & 0.9938 & 0.9049 & 0.9524 & 0.9497 \\
7 & 0.6490 & 0.9954 & 0.9114 & 0.9490 & 0.9473 \\
8 & 0.5521 & 0.9993 & 0.9682 & 0.9311 & 0.9419 \\
9 & 0.5791 & 0.9912 & 0.8536 & 0.9639 & 0.9578 \\
10 & 0.6343 & 0.9877 & 0.8225 & 0.9678 & 0.9586 \\
11 & 0.5443 & 0.9972 & 0.9419 & 0.9489 & 0.9486 \\
12 & 0.6093 & 0.9972 & 0.9308 & 0.9454 & 0.9448 \\
13 & 0.4632 & 0.9975 & 0.9525 & 0.9449 & 0.9452 \\
14 & 0.6410 & 0.9879 & 0.8236 & 0.9690 & 0.9598 \\
15 & 0.5530 & 0.9942 & 0.8817 & 0.9665 & 0.9627 \\
16 & 0.6586 & 0.9896 & 0.8633 & 0.9669 & 0.9597 \\
17 & 0.6066 & 0.9905 & 0.8599 & 0.9646 & 0.9581 \\
18 & 0.6861 & 0.9881 & 0.8329 & 0.9733 & 0.9642 \\
19 & 0.7412 & 0.9910 & 0.8818 & 0.9769 & 0.9702 \\
20 & 0.5997 & 0.9913 & 0.8468 & 0.9689 & 0.9625 \\
Average & $\mathbf{0 . 6 2 8 9}$ & $\mathbf{0 . 9 9 2 4}$ & $\mathbf{0 . 8 8 7 5}$ & $\mathbf{0 . 9 5 7 8}$ & $\mathbf{0 . 9 5 4 1}$ \\
\hline
\end{tabular}

Table 2: Performance evaluation on the DRIVE database 


\begin{tabular}{|cccccc|}
\hline Image & $S e$ & $S p$ & $P P V$ & $N P V$ & $A c c$ \\
\hline 1 & 0.6433 & 0.9893 & 0.8739 & 0.9603 & 0.9537 \\
2 & 0.5845 & 0.9946 & 0.9238 & 0.9554 & 0.9534 \\
3 & 0.5687 & 0.9892 & 0.8631 & 0.9504 & 0.9443 \\
4 & 0.7123 & 0.9831 & 0.8163 & 0.9700 & 0.9572 \\
5 & 0.7260 & 0.9868 & 0.8477 & 0.9727 & 0.9629 \\
6 & 0.7975 & 0.9742 & 0.7802 & 0.9767 & 0.9560 \\
7 & 0.7177 & 0.9870 & 0.8460 & 0.9724 & 0.9627 \\
8 & 0.8001 & 0.9741 & 0.7779 & 0.9773 & 0.9565 \\
9 & 0.5989 & 0.9915 & 0.8586 & 0.9665 & 0.9606 \\
10 & 0.7112 & 0.979 & 0.7646 & 0.9725 & 0.9557 \\
11 & 0.7936 & 0.9798 & 0.8039 & 0.9785 & 0.9662 \\
12 & 0.8226 & 0.9765 & 0.8058 & 0.9789 & 0.9602 \\
13 & 0.7663 & 0.9751 & 0.7479 & 0.9771 & 0.9565 \\
14 & 0.8062 & 0.9597 & 0.6590 & 0.9808 & 0.9462 \\
15 & 0.8413 & 0.9706 & 0.7113 & 0.9861 & 0.9604 \\
Average & $\mathbf{0 . 7 2 6 0}$ & $\mathbf{0 . 9 8 0 7}$ & $\mathbf{0 . 8 0 5 3}$ & $\mathbf{0 . 9 7 1 7}$ & $\mathbf{0 . 9 5 6 8}$ \\
\hline
\end{tabular}

Table 3: Performance evaluation on the healthy group

(HRF)

\begin{tabular}{|cccccc|}
\hline Image & $S e$ & $S p$ & $P P V$ & $N P V$ & $A c c$ \\
\hline 1 & 0.8048 & 0.9700 & 0.5796 & 0.9897 & 0.9620 \\
2 & 0.7697 & 0.9719 & 0.6416 & 0.9847 & 0.9595 \\
3 & 0.7873 & 0.9600 & 0.5487 & 0.9865 & 0.9499 \\
4 & 0.7490 & 0.9696 & 0.5844 & 0.9854 & 0.9577 \\
5 & 0.8592 & 0.9664 & 0.618 & 0.9908 & 0.9600 \\
6 & 0.5068 & 0.9807 & 0.6728 & 0.9621 & 0.9462 \\
7 & 0.8463 & 0.9594 & 0.6311 & 0.9870 & 0.9508 \\
8 & 0.8482 & 0.9492 & 0.5691 & 0.9875 & 0.9417 \\
9 & 0.5626 & 0.9851 & 0.729 & 0.9693 & 0.9570 \\
10 & 0.6800 & 0.9798 & 0.7628 & 0.9698 & 0.9537 \\
11 & 0.8107 & 0.9646 & 0.6761 & 0.9824 & 0.9518 \\
12 & 0.8777 & 0.9291 & 0.4628 & 0.9909 & 0.9258 \\
13 & 0.8143 & 0.9656 & 0.6295 & 0.9864 & 0.9555 \\
14 & 0.8505 & 0.9285 & 0.6848 & 0.9873 & 0.9227 \\
15 & 0.8664 & 0.9274 & 0.6944 & 0.9903 & 0.9235 \\
Average & $\mathbf{0 . 7 7 5 5}$ & $\mathbf{0 . 9 6 0 4}$ & $\mathbf{0 . 6 3 2 3}$ & $\mathbf{0 . 9 8 3 3}$ & $\mathbf{0 . 9 4 7 8}$ \\
\hline
\end{tabular}

Table 4: Performance evaluation on the DR Group (HRF) 


\begin{tabular}{|cccccc|}
\hline Image & $S e$ & $S p$ & $P P V$ & $N P V$ & $A c c$ \\
\hline 1 & 0.6699 & 0.9869 & 0.7757 & 0.9779 & 0.9669 \\
2 & 0.8426 & 0.9654 & 0.6994 & 0.9877 & 0.9567 \\
3 & 0.5544 & 0.9958 & 0.8639 & 0.9681 & 0.9651 \\
4 & 0.8001 & 0.9733 & 0.6664 & 0.9865 & 0.9625 \\
5 & 0.7505 & 0.9850 & 0.7733 & 0.9831 & 0.9701 \\
6 & 0.7620 & 0.9801 & 0.7307 & 0.9831 & 0.9657 \\
7 & 0.7177 & 0.9859 & 0.7726 & 0.9812 & 0.9691 \\
8 & 0.8563 & 0.9614 & 0.6067 & 0.9897 & 0.9546 \\
9 & 0.7455 & 0.9818 & 0.7364 & 0.9826 & 0.9667 \\
10 & 0.7385 & 0.9809 & 0.7243 & 0.9822 & 0.9655 \\
11 & 0.7334 & 0.9799 & 0.7465 & 0.9785 & 0.9615 \\
12 & 0.8052 & 0.9669 & 0.6822 & 0.9825 & 0.9538 \\
13 & 0.7450 & 0.9739 & 0.6731 & 0.9815 & 0.9586 \\
14 & 0.7804 & 0.9581 & 0.5856 & 0.9829 & 0.9456 \\
15 & 0.5013 & 0.9927 & 0.8411 & 0.9628 & 0.9576 \\
Average & $\mathbf{0 . 7 2 6 0}$ & $\mathbf{0 . 9 8 0 7}$ & $\mathbf{0 . 8 0 5 3}$ & $\mathbf{0 . 9 7 1 7}$ & $\mathbf{0 . 9 5 6 8}$ \\
\hline
\end{tabular}

Table 5: Performance evaluation on the Glaucomatous Group (HRF)

\begin{tabular}{|lccc|}
\hline Method & $A c c$ & $S e$ & $S p$ \\
\hline Zhao et al. [1] & 0.9477 & 0.7354 & 0.9789 \\
Zhang et al. [5] & 0.9382 & 0.7120 & 0.9724 \\
Cinsdikici et al. [6] & 0.9293 & - & - \\
Budai et al. [9] & 0.9572 & 0.6440 & 0.9870 \\
Azzopardi et al. [10] & 0.9442 & 0.7655 & 0.9704 \\
Soares et al. [14] & 0.9466 & - & - \\
Xinge You et al. [31] & 0.9434 & 0.7410 & 0.9751 \\
Odstrcilik et al. [32] & 0.9473 & 0.7807 & 0.9712 \\
Proposed Method & $\mathbf{0 . 9 5 4 1}$ & $\mathbf{0 . 6 2 8 9}$ & $\mathbf{0 . 9 9 2 4}$ \\
\hline
\end{tabular}

Table 6: Segmentation performance comparisons of different methods on the DRIVE database

\begin{tabular}{|clccc|}
\hline Performance & \multicolumn{1}{c}{ Methods } & Healthy Images & DR Images & Glaucomatous Images \\
\hline \multirow{3}{*}{ Acc } & Budai et al. [9] & 0.961 & 0.955 & 0.965 \\
& Odstrcilik et al. [32] & 0.953 & 0.944 & 0.949 \\
& Frangi et al. [33] & 0.955 & 0.946 & 0.961 \\
& Proposed Method & $\mathbf{0 . 9 5 6}$ & $\mathbf{0 . 9 4 8}$ & $\mathbf{0 . 9 6 1}$ \\
\hline \multirow{3}{*}{$S e$} & Budai et al. [9] & 0.662 & 0.658 & 0.687 \\
& Odstrcilik et al. [32] & 0.786 & 0.746 & 0.790 \\
& Frangi et al. [33] & 0.621 & 0.590 & 0.654 \\
& Proposed Method & $\mathbf{0 . 7 2 6}$ & $\mathbf{0 . 7 7 5}$ & $\mathbf{0 . 7 3 3}$ \\
\hline \multirow{3}{*}{$S n$} & Budai et al. [9] & 0.992 & 0.975 & 0.993 \\
& Odstrcilik et al. [32] & 0.977 & 0.9619 & 0.978 \\
& Frangi et al. [33] & 0.989 & 0.972 & 0.984 \\
& Proposed Method & $\mathbf{0 . 9 8 0}$ & $\mathbf{0 . 9 6 0}$ & $\mathbf{0 . 9 7 7}$ \\
\hline
\end{tabular}

Table 7: Segmentation performance comparisons of different methods on the HRF database 


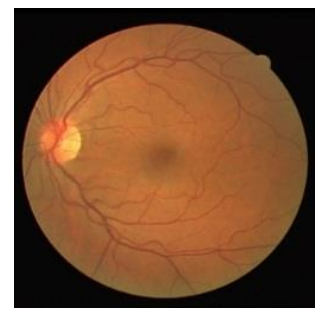

(a)

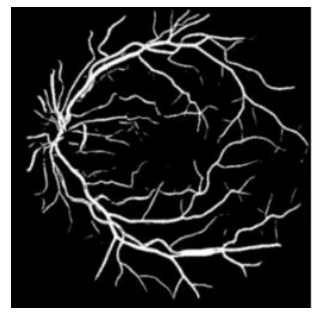

(d)

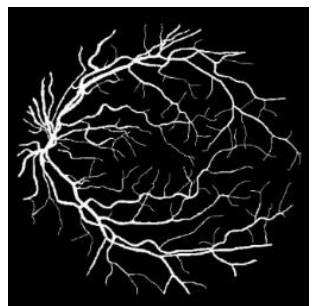

(b)

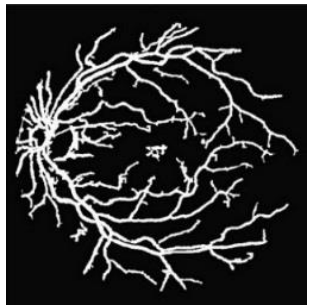

(e)

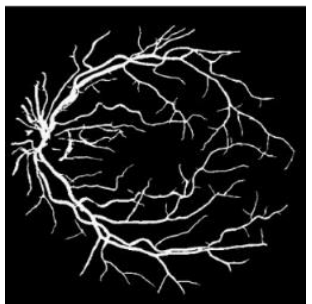

(c)

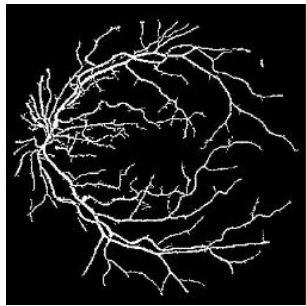

(f)

Figure 7: Segmentation results of first retinal image from the DRIVE database using different methods (a) Original image, (b) ground truth image of the first observer, (c) Qian et al. [1] , (d) Soares et al. [15], (e) Cinsdikici et al. [7] and (f) proposed method.

\section{Conclusion}

Exact segmentation of retinal blood vessels plays a principal role for identification and cure of various pathological syndromes. In this paper we introduced an automatic segmentation method for segmentation of retinal blood vessels in ophthalmoscope images based on Gabor wavelet and morphological reconstruction approach. The Gabor wavelet is used to enhance the vascular pattern of retinal blood vessels while morphological reconstruction approach extract the retinal blood vessels. The morphological reconstruction approach revealed that it is a very useful technique for extraction of retinal blood vessels without destruction of any small details.

The performance of the proposed method is analyzed by comparing the obtained segmented output with the gold standard image and is evaluated on the publicly available digital retinal images for vessel extraction (DRIVE) and high resolution fundus (HRF) images database. The performance of the proposed method is compared with other methods from which we can observed that our method outperforms the other methods reaching an average accuracies of 0.9531 on the DRIVE database and $0.9569,0.9478$, and 0.9613 on the HRF (images of healthy, diabetic retinopathy and glaucomatous retinas) database respectively. The proposed method performs well in maintaining connectivity between the retinal vessels and easier to implement. The main pitfall is that it gives less sensitivity, as in some pathological images, some structure are misclassified as vessels which gives poor segmentation result. We will further examines this aspects in our upcoming work.

\section{References}

[1] Y.Q. Zhao, X.H. Wang, X.F. Wang, F.Y. Shih, "Retinal vessels segmentation based on level set and region growing", Pattern Recognition, 47 (3): 2437-2446, 2014. DOI: 10.1016/j.patcog.2014.01.006

[2] G. Matsopoulos, P. Asvestas, K. Delibasis, N. Mouravliansky, T. Zeyen, "Detection of glaucomatous change based on vessel shape analysis", Med Imaging Graphics, 30: 139-145, 2006. DOI:10.1016/j.compmedimag.2007.11.003 
[3] S. Chaudhuri, S. Chatterjee, N. Katz, M. Nelson, M. Goldbaum, "Detection of blood vessels in retinal images using two dimensional matched filters", IEEE Transactions on Medical Imaging, 8 (3): 263-269, 1989. DOI: $10.1109 / 42.34715$

[4] M. Al-Rawi, H. Karajeh, "Genetic algorithm matched filter optimization for automated detection of blood vessels from digital retinal images", Computer Methods and Programs in Biomedicine, 87 (3): 248-253, 2007. DOI:10.1016/j.cmpb.2007.05.012

[5] Bob Zhang, Lin Zhang, Lei Zhang, Fakhri Karray, "Retinal vessel extraction by matched filter with first-order derivative of Gaussian", Computers in Biology and Medicine, 40 (4): 438-445, 2010. DOI:10.1016/j.compbiomed.2010.02.008

[6] M.G. Cinsdikici, D. Aydın, "Detection of blood vessels in ophthalmoscope images using MF / ant (matched filter / ant colony) algorithm", Computer Methods and Programs in Biomedicine, 96 (2): 85-95, 2009. DOI:10.1016/j.cmpb.2009.04.005

[7] K.S. Sreejini, V.K. Govindan, "Improved multiscale matched filter for retina vessel segmentation using PSO algorithm", Egyptian Informatics Journal, 16 (3): 253-260, 2015. DOI: org/10.1016/j.eij.2015.06.004

[8] F. Rossant, M. Badellino, A. Chavillon, I. Bloch, M. Paques, "A morphological approach for vessel segmentation in eye fundus images, with quantitative evaluation", Journal of Medical Imaging and Health Informatics, 1 (1): 42-49, 2011. DOI:10.1166/jmihi.2011.1006

[9] A. Budai, R. Bock, A. Maier, J. Hornegger, G. Michelson, "Robust vessel segmentation in fundus images", International Journal of Biomedical Imaging, 1-11, 2013. DOI: 10.1155/2013/154860

[10] G. Azzopardi, N. Strisciuglio, M. Vento, N. Petkov, "Trainable COSFIRE filters for vessel delineation with application to retinal images", Medical Image Analysis, 19: 46-57, 2015. DOI: 10.1016/j.media.2014.08.002

[11] M.M. Fraz, P. Remagnino, A. Hoppe, B. Uyyanonvara, A.R. Rudnicka, C.G. Owen, S.A. Barman, "Blood vessel segmentation methodologies in retinal images - A survey", Computer Methods and Programs in Biomedicine, 108 (1): 407-433, 2011. DOI: 10.1016/j.cmpb.2012.03.009

[12] A. Can, H. Shen, J.N. Turner, H.L. Tanenbaum, B. Roysam, "Rapid automated tracing and feature extraction from retinal fundus images using direct exploratory algorithms", IEEE Transactions on Information Technology in Biomedicine, 3 (2): 125-138, 1999. DOI: 10.1109/4233.767088

[13] K.K. Delibasis, A.I. Kechriniotis, C. Tsonos, N. Assimakis, "Automatic model-based tracing algorithm for vessel segmentation and diameter estimation", Computer Methods and Programs in Biomedicine, 100 (2): 108-122, 2010. DOI:10.1016/j.cmpb.2010.03.004

[14] M. Mohri, A. Rostamizadeh, A. Talwalkar, Foundations of machine learning, MIT Press ,2012.

[15] J.V. B. Soares, J.J. G. Leandro, R.M. Cesar Jr., H.F. Jelinek, M.J. Cree, "Retinal Vessel Segmentation Using the 2-D Gabor Wavelet and Supervised Classification”, IEEE Transactions on Medical Imaging, 25 (9): 1214-1222, 2006. DOI: 10.1109/TMI.2006.879967

[16] J. Staal, M.D. Abràmoff, M. Niemeijer, M.A. Viergever, B. Ginneken, "Ridge-based vessel segmentation in color images of the retina", IEEE Transactions on Medical Imaging, 23 (4): 501-509, 2004. DOI: 10.1109/TMI.2004.825627

[17] G.B. Kande, P.V. Subbaiah, T.S. Savithri, "Unsupervised fuzzy based vessel segmentation in pathological digital fundus images", Journal of Medical Systems, 34 (5): 849-858, 2010. DOI: 10.1007/s10916-009-92990

[18] Zuiderveld, Karel, Contrast Limited Adaptive Histograph Equalization, Graphic Gems IV. San Diego, Academic Press Professional, 474-485, 1994.

[19] S. M. Pizer, E. P. Amburn, J. D. Austin, "Adaptive histogram equalization and its variations", Computer Vision, Graphics, and Image Processing, 39: 355-368, 1987.

[20] I. Fogel, D. Sagi, “Gabor filter as texture discriminator”, Biological Cybernetics, 61 (2): 103-113, 1989. DOI: 10.1007/BF00204594 
[21] J. Dash, N. Bhoi, "a survey on blood vessel detection methodologies in retinal images", Computational Intelligence and Network (CINE), International Conference on IEEE, KIIT, India, 166-171, 2015. DOI: 10.1109/CINE.2015.39

[22] L. Shen, L. Bai, “A review on Gabor wavelets for face recognition”, Pattern Analysis and Applications, 9 (2): 273-292, 2006. DOI: 10.1007/s10044-006-0033-y

[23] M.U. Akram, A. Tariq, S.A. Khan, "Retinal Image Blood Vessel Segmentation", International Conference on Information and Communication Technology (ICICT'09): 181-192, $2009 . \quad$ DOI: 10.1109/ICICT.2009.5267194

[24] M.U. Akram, I. Jamal, A. Tariq, "Blood vessel enhancement and segmentation for screening of diabetic retinopathy", Telecommunication Computing Electronics and Control, 10(2): 327-334, 2012. DOI: 10.11591/telkomnika.v10i2.686

[25] R.C. Gonzalez, R.E. Woods, Digital Image Processing, Prentice Hall 2nd edition, 2002.

[26] Pierre Soille, Morphological Image Analysis Principles and Applications, Springer Berlin Heidelberg 2nd edition, 2004.

[27] C. Heneghan, J. Flynn, M. Keefe, M. Cahill, "Characterization of changes in blood vessel width and tortuosity in retinopathy of prematurity using image analysis", Medical Image Analysis, 6 (4): 407-429, 2002. DOI:10.1016/j.patcog.2011.01.007

[28] D. Marín, A. Aquino, M.E. Gegúndez-Arias, J.M. Bravo, "A new supervised method for blood vessel segmentation in retinal images by using gray-level and moment invariants-based features", IEEE Transactions on Medical Imaging, 30 (1): 146-158, 2011. DOI: 10.1109/TMI.2010.2064333

[29] M. Niemeijer, J. J. Staal, B. V. Ginneken, M. Loog, M.D. Abramoff, "DRIVE, digital retinal images for vessel extraction”, http://www.isi.uu.nl/Research/Databases/DRIVE, (2004).

[30] A. Budai, J. Odstricilik, R. Kollar, J. Jan, T. Kubena, G. Michelson, "A public database for the evaluation of fundus image segmentation algorithms", https://www5.cs.fau.de/research/data/fundus-images.

[31] X. You, Q. Peng, Y. Yuan, Y. Cheung, J. Lei, "Segmentation of retinal blood vessels using the radial projection and semi-supervised approach", Pattern Recognition, 44 (10-11): 2314-2324, 2011. DOI:10.1016/j.patcog.2011.01.007

[32] J. Odstrcilik, R. Kolar, A. Budai, J. Hornegger, J. Jan, J. Gazarek, T. Kubena, P. Cernosek, O. Svoboda, E. Angelopoulou, "Retinal vessel segmentation by improved matched filtering: evaluation on a new highresolution fundus image database", IET Image Processing, 7 (4): 373-383, 2013. DOI: 10.1049/ietipr.2012.0455

[33] A.F. Frangi. J. Niessen, K.L. Vincken, M.A. Viergever, Multiscale Vessel Enhancement Filtering, Springer, Heidelberg, Germany, 1998.

[34] T. Mapayi, S. Viriri, J. Tapamo, "Comparative study of retinal vessel segmentation based on global thresholding techniques", Computational and Mathematical Methods in Medicine, 1-14, 2015. DOI: $10.1155 / 2015 / 895267$. 